\title{
THE ECONOMICS OF TRAVELER INFORMATION FROM PROBES
}

\author{
DAVID LEVINSON \\ University of Minnesota
}

\begin{abstract}
This article examines the economics of traveler information from probe vehicles to understand how many probes are needed to provide useful information and how that probe information might be supplied to travelers. Probes differ from permanently installed roadway detection devices both because they provide information that is less current and because an information system centered on this technology can be organized in the form of private clubs rather than a government agency. This article estimates travel time associated with various shares of probes among the fleet by simulating different levels of probes, information subscription, and congestion. It examines the travel time saving under both recurring and nonrecurring congestion. With the latter, a low frequency of probes is sufficient to detect the incident and enable information consumers to choose alternates. However, smoothing the stochastic nature of traffic under recurring congestion requires a relatively high share of probes, depending on the level of congestion.
\end{abstract}

$\mathrm{W}$ hy isn't an accurate traveler information system that tells commuters the fastest route to their destination in real time widely deployed? The component technologies to provide this information have been tested for more than a decade. Modern computers and software can very quickly find the shortest route between two points given the travel times on each link. Communications systems can transmit the request for information and the result between a mobile phone and a base station. Devices can display that information in the vehicle.

One significant problem is the lack of data; most transportation agencies still cannot tell users the travel speed on each segment of the freeway network, much less the arterial system. Although many technologies (loop detectors, video cameras, lasers, radar guns) can measure spot speeds, from which link speeds and travel times can be estimated, none are sufficiently cheap and reliable and none have been installed ubiquitously.

An institutional economist might argue that the information is not available because the incentive structure is wrong. Public highway agencies have no explicit incentive to reduce travelers' time or increase the number of users. There is no reward for efficiency; it may even lead to less construction, less staff, and less budget. That suggests considering alternative sources and institutional arrangements for how an information provider obtains its information. There are alternatives to the conventional answer of publicly controlled permanent monitoring stations on the freeway (cameras, loop detectors, etc.).

This article considers one approach in which consumers of travel information become producers as well by acting as probes to update travel times for an information provider and thus provide accurate information for following roadway consumers. This approach has been undertaken in San Antonio, Texas, as part of the Transguide program (Dallenback \& Fariello, 2001).

PUBLIC WORKS MANAGEMENT \& POLICY, Vol. 6 No. 4, April 2002 241-249

(C) 2002 Sage Publications
David Levinson teaches transportation engineering at the University of Minnesota. His research interests are in network evaluation, pricing, and modeling. He has recently completed the book Financing Transportation Networks.

\author{
Modern computers and \\ software can very \\ quickly find the shortest \\ route between two \\ points given the travel \\ times on each link.
}


Table 1: Summary of Time Saved With Advanced Traveler Information Systems by Study

\begin{tabular}{|c|c|c|c|}
\hline Author & Congestion Level/Type & Time Saved (\%) & Market Share (\%) \\
\hline \multirow[t]{2}{*}{ Adler, Blue, and Wu (1999) } & Free flow $(800 \mathrm{vph})$ & 2.7 & 100 \\
\hline & Congestion (1500 vph) & 3.1 & 80 \\
\hline \multirow[t]{3}{*}{ Wunderlich (1996) } & Rain ( $25 \%$ drop in overall capacity) & 12 & 10 \\
\hline & Construction (50\% drop in capacity locally) & 18 & 10 \\
\hline & Incident (50\% drop in capacity locally) & 6 & 10 \\
\hline Emmerink (1996) & Recurrent congestion & 1 to 4 & 100 \\
\hline \multicolumn{4}{|l|}{ Emmerink, Axhausen, and } \\
\hline Rietveld (1995) & Recurrent congestion & 7 & \\
\hline \multirow[t]{2}{*}{ Wunderlich (1995a) } & Congestion but not reaching saturation & 8 to 20 & 5 \\
\hline & Saturation & 7 to 12 & 5 \\
\hline Wunderlich (1996) & Capacity-reducing incident & 15 & \\
\hline Al-Deek and Kanafani (1989) & Difference in SO and UE & 3 to 4 & \\
\hline \multirow[t]{2}{*}{ Al-Deek and Kanafani (1993) } & Modeling of incident conditions & 30 to 40 & \\
\hline & & 25 to 55 & \\
\hline $\begin{array}{l}\text { STORM, Stuttgart, Germany } \\
\text { (Peckmann, 1996) }\end{array}$ & Field Operational Test Simulation & 20 & \\
\hline \multirow{3}{*}{$\begin{array}{l}\text { TravTek, Orlando, Florida, } \\
1998 \text { (U.S. Department of } \\
\text { Transportation, 1995) }\end{array}$} & Field Operational Test Simulation & 5 & 10 \\
\hline & & 8 & 30 \\
\hline & & 11 & 50 \\
\hline
\end{tabular}

SOURCE: Levinson, Gillen, and Chang (1999).

NOTE: $\mathrm{vph}=$ vehicles per hour; $\mathrm{SO}=$ system optimal; $\mathrm{UE}=$ user equilibrium. Time saved overall or equipped vehicles only.

Probes are becoming increasingly technically feasible with the prevalence of new generation mobile telephones with automatic location technologies, such as a global positioning system that provides continuous tracking of vehicles. Alternative technologies, such as the transponders used for electronic toll collection, allow point-to-point tracking of vehicles. Either continuous or point-to-point technologies can be adapted to provide traffic information. More probe vehicles imply more recent and accurate information. The information provided remains within a network, or club, of probe vehicles and information consumers so that the larger the club, the more valuable it is. Although there may be multiple sustainable clubs (and diminishing marginal returns to club size), small clubs are less valuable than large ones.

The disadvantage of probes is that roadway coverage is still incomplete. A low share of probes results in insufficiently fresh information, which may be unable to be used to smooth out the daily random variations in traffic. Conditions may change between the time information is collected and the time it is presented to drivers. Several drivers will possess the same dated facts and act incorrectly on them. This article will estimate the coverage levels necessary for positive outcomes for both recurring and nonrecurring congestion.

Table 1 summarizes the results of some recent studies of Advanced Traveler Information Systems. These results provide a baseline to compare the results from studies using probes, such as this one. Boyce, Hicks, and Sen (1991) used simulation to estimate the number of vehicle probes needed to monitor link travel times adequately during peak periods, concluding that about 4,700 vehicle probes are needed to traverse $50 \%$ of all arterial links every 5 minutes. Rakha and Van Aerde (1995) assessed the accuracy of vehicle probe estimates using a smallscale field test in Orlando, Florida, finding that vehicle probes underestimated the trip duration for all of the nine test runs, with an average error of about $-12 \%$ and a standard deviation of approximately 7\%. Sen, Thakuriah, Zhu, and Karr (1997) used data from the suburban Chicago ADVANCE project during the summer of 1995 to estimate the number of probes per unit time required to measure the mean travel time, finding that a high level of deployment does not improve guidance quality substantially. Saricks, Shofer, Sööt, and Belella (1997) also evaluated the ADVANCE project, incorporating the data gathered into a historic average link travel time database (by time of day) to provide the shortest time route to drivers, finding that when com- 
Table 2: Defining Public, Private, Club and "Congesting” Goods

\begin{tabular}{llll}
\hline & & \multicolumn{2}{c}{ Excludability } \\
\cline { 3 - 4 } & & \multicolumn{1}{c}{ Yes } & \multicolumn{1}{c}{ No } \\
\hline Rivalry & Yes & Private & "Congesting” \\
& No & Club & Public \\
\hline
\end{tabular}

pared to manually recorded travel data, $87.6 \%$ of probe values were within $\pm 5 \mathrm{sec}$ and $90 \%$ were within $\pm 10 \mathrm{sec}$.

First, we consider the issue of who provides Advanced Traveler Information Systems. Then we develop a simulation model to analyze the effectiveness of varying levels of probes under differing congestion conditions. The model is then applied, and results are compared for a variety of cases. We summarize our results and conclude with some thoughts about sources of realtime traffic information.

\section{Information Providers}

The provider of a traveler information service is inextricably linked with the objective used to decide how to disseminate that information. The appropriateness of the provider further depends on whether traveler information is a public or private good. Two criteria can help classify a good as public or private: excludability and rivalry. Excludability implies that the good's provider can prevent a user from obtaining it without charge. Rivalry implies that one person's consumption of a particular good prevents another individual from consuming it. Table 2 summarizes goods by type.

Traveler information is potentially excludable assuming some sort of encrypted or scrambled communication system between information provider and information consumer. The ability of the public sector to exclude citizens from travel data collected with public funds may have legal and political ramifications (although the government often charges for documents and other value added services), but it is not technologically impossible. However, the private sector would easily be able to exclude consumers from data collected with private funding.

Information is in some senses nonrivalrous; one person's possession of information does not prevent another from having it. However, one individual's possession of information may diminish its value for another. This issue will be investigated with the traffic simulation. Scarce or unique information can be more valuable to its possessor than common knowledge. The information itself may be nonrivalrous, but the production of information is less so; there are limited computer resources; and generating the information has some costs, for instance, developing the route for Consumer A may delay the computation for Consumer B. Nevertheless, collecting, processing, and transmitting information all have a relatively high fixed cost and a low (and not necessarily rising) variable cost. An infrastructure to collect, process, and distribute information must be constructed, but once constructed, adding an additional user is relatively inexpensive.

Traveler information's excludability and nonrivalry most closely resembles a club good. Club goods can be provided successfully by the private or the intermediate (nonprofit) sectors. They do not require government provision, although many club goods are publicly provided. ${ }^{1}$

\section{Modeling Approach}

Additional users of the transportation system are generally thought of as producing negative externalities: Drivers impose congestion on those who follow. An information network, on the other hand, becomes more valuable the more users it has providing real-time data. Those same drivers who cause congestion also can inform those behind of traffic conditions. This informa-
Then we develop a simulation model to analyze the effectiveness of varying levels of probes under differing congestion conditions.

\section{Scarce or unique information can be more valuable to its possessor than common knowledge.}




\section{Travel distances are ignored, and the queue is treated as an infinite capacity point queue.}

tion might encourage the following drivers to change their anticipated route and lower their travel time. Care of course must be given to ensure that providing information does not simply relocate the problem, if everyone follows the same advice. The more links that are covered by drivers in an information network, the greater the quantity, freshness, and accuracy of the information. The model presented here shows the usefulness of various levels of probe coverage.

Modeling the effect of information from probes requires use of a simulation that probabilistically varies approach flow (we assume Poisson arrivals), the road's service rate, and the appearance of probes, which is random based on the share of probes in the fleet. Our analysis assumes a simple two parallel link network. ${ }^{2}$ A driver who has an information system in his vehicle is labeled informed; one who does not is called uninformed. Because of queuing externalities, both informed and uninformed drivers are affected by information. Informed drivers receive their information before deciding which of the two links to take. While the average headway (the time between vehicle $v$ and $v-1$ ) is simply $1 / \lambda$, each vehicle's simulated arrival headway $(H v)$ in seconds is given by the following expression:

$$
\mathrm{Hv}=-\mathrm{LN}(\mathrm{RAND}()) / \lambda
$$

Where $R A N D()=$ indicates a random real number between 0 and 1 and $\lambda=$ average arrival rate in vehicles per second.

The expected travel time on each link for informed drivers is simply the length of the queue on the link at the time of arrival at the fork in the road (the decision point) multiplied by the average service rate. The expected time for uninformed drivers is based on history, but because of symmetry in service rates, we assume that it is the same for each link. Thus, uninformed drivers are equally probable on either link. Travel distances are ignored, and the queue is treated as an infinite capacity point queue. The average service rate is adjusted in the case of incidents as discussed below. The process is illustrated in Figure 1. The simulated service time $(\tau)$ in seconds per vehicle is given by the equation below:

$$
\tau_{v}=\tau+\sigma^{*}(\operatorname{RAND}()-0.5)
$$

where $\tau_{v}=$ service time for vehicle $v$ and $\tau=$ average service time in seconds, assumed to be 2 seconds unless otherwise given $\sigma=$ service time range (sec), assumed to be 0.5 seconds. (This random number is distinct from that in the previous equation). This equation means that the service time for vehicle $v$ is equal to a fixed (average) component $\tau$ plus a random component, which is between plus or minus $0.5^{*} \sigma$. Given the assumed values $\tau=2$ seconds and $\sigma=0.5$ second, this means that service times vary smoothly and randomly between 1.75 and 2.25 seconds.

\section{Modeling Results}

This section examines how congestion levels vary with how many drivers use Advanced Traveler Information Systems and what percentage of the fleet is comprised of probes. The percentage of probes is important, because if only a few vehicles are probes, information will no longer be fresh. That is particularly pertinent when trying to save time from a stochastic process, such as recurring congestion, because every second counts. It is likely to be less severe in the case of nonrecurring congestion, which takes longer to clear. It is important to be able to identify what percentage of the fleet needs to be probes to save time, because this will affect the cost of the system. Another advantage of probes is that they could be implemented with less government involvement because they do not necessarily rely on installing devices on the public rightof-way. However, if probes are the only source of information, problems can arise when information is no longer recent; misinformation can be worse than no information. This suggests that probes require a large critical mass to be successful or that they are best used in combination with other sources. However, this section assumes that only probes provide information in order 


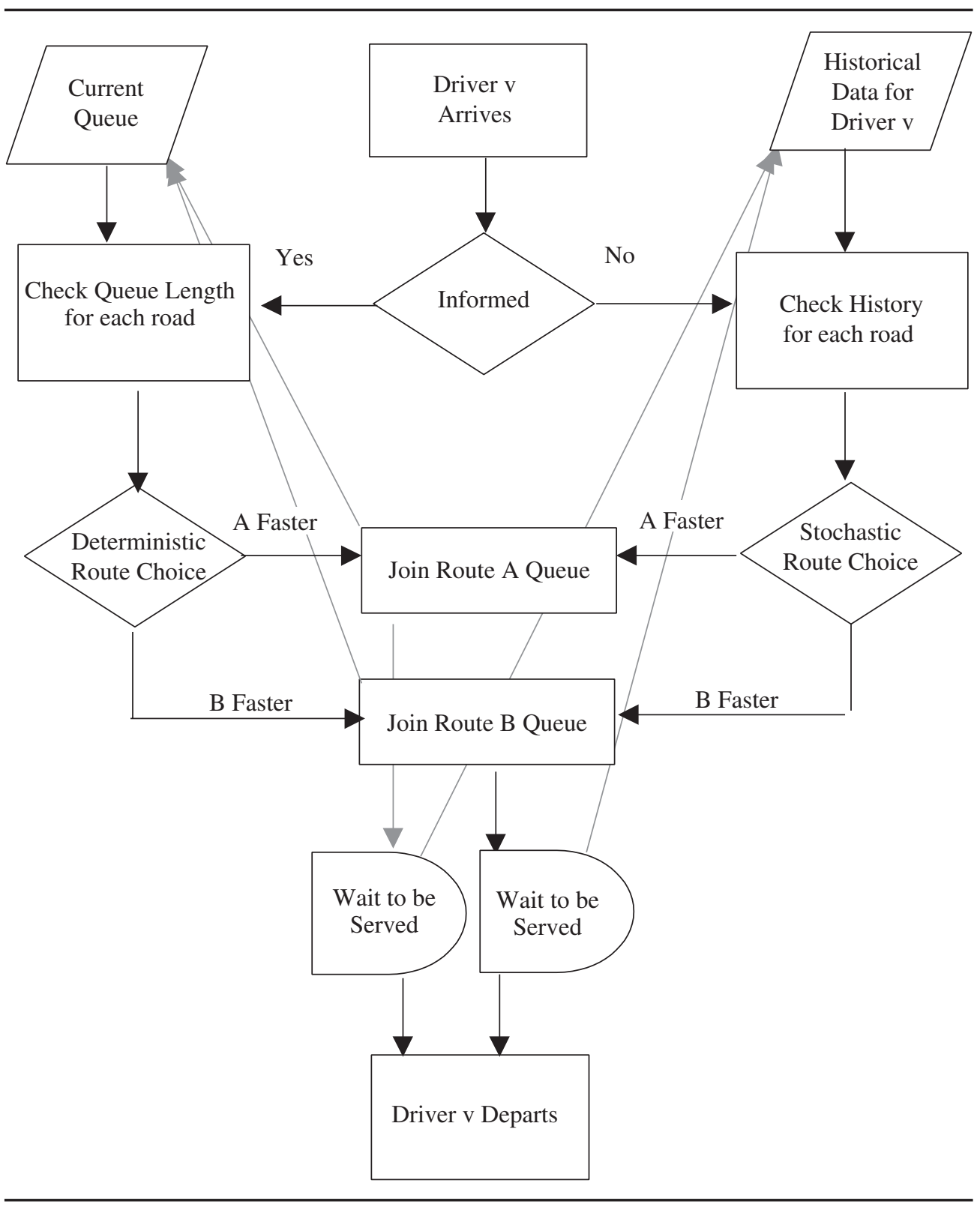

Figure 1: Flowchart of Modeling Methodology

to determine where that critical mass arises. Clearly, intelligent combination of probe data with other sources may have advantages over using either source independently.

\section{RECURRING CONGESTION}

Figure 2 shows the average travel time for informed drivers as the congestion level and the percentage of probes on the network increases from $0 \%$ to $100 \%$. Figure 3 shows that result for the uninformed travelers. In these cases, the percentage informed averages 50\%, although samples are drawn equally from 30 runs each at $0 \%, 17 \%, 33 \%, 50 \%, 67 \%, 83 \%$, and $100 \%$. Informed drivers are best off if the information is not widely distributed, because they can shift routes and not have to compete with others shifting routes. However, uninformed drivers do benefit from wide distribution of information to others, because this moves the informed travel-

Informed drivers are best off if the information is not widely distributed, because they can shift routes and not have to compete with others shifting routes. 


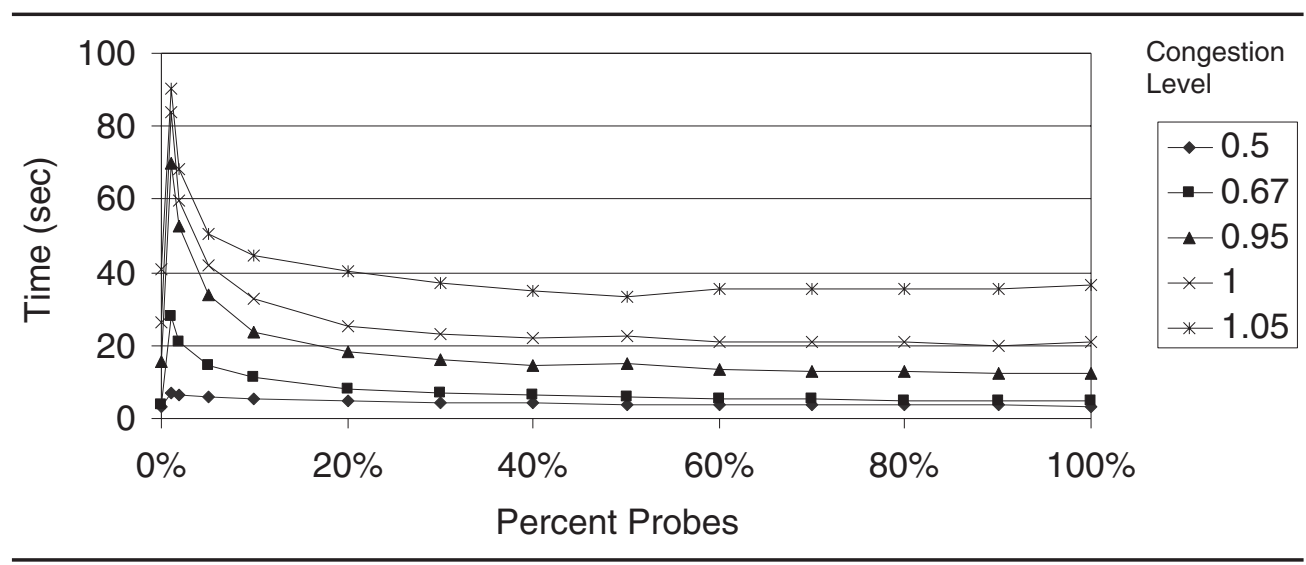

Figure 2: Average Time (Informed Travelers) by Percentage Probes and Congestion Levels

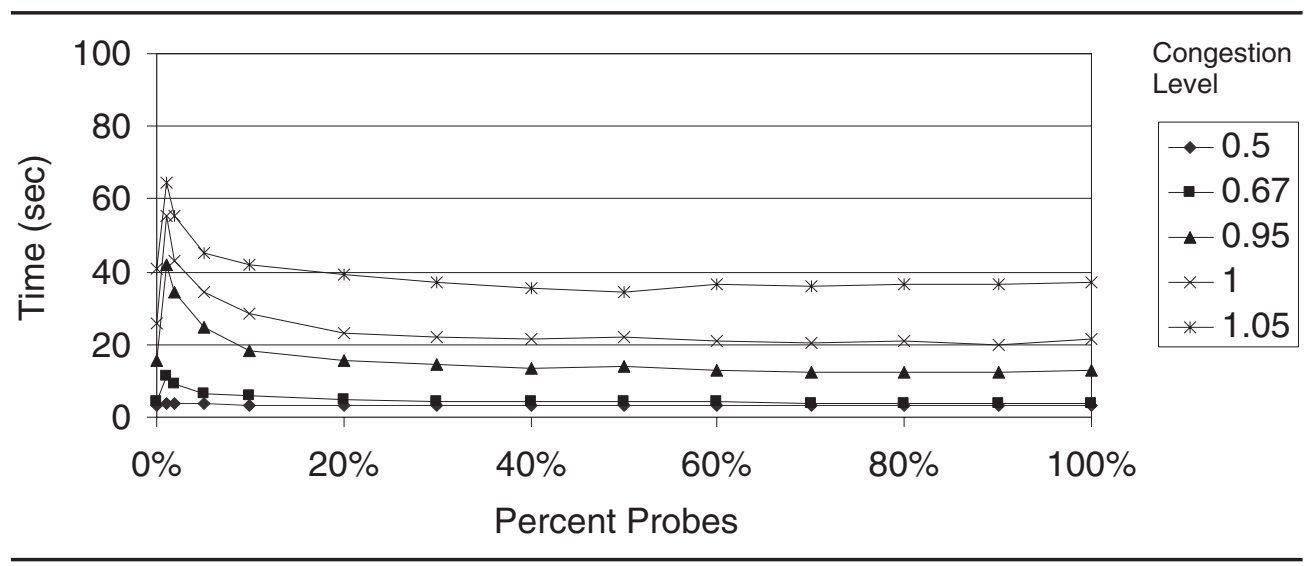

Figure 3: Average Time (Uninformed Travelers) by Percentage Probes and Congestion Levels

ers from the more to the less congested routes, so the uninformed drivers on the more congested routes have fewer other vehicles to contend with. Of course, uninformed drivers on the formerly less congested route have more vehicles to contend with, but the net effect is to improve travel times overall. Informed drivers do better than uninformed drivers, assuming the information is accurate. All together, information is a good thing for reducing congestion. Note that the benefits of probes are steadily increasing. If there are few probes, the average travel time is high compared with many probes (listening to old information is counterproductive). It is also important to note that probelike information (i.e., information with lags) is most useful at or near congestion for cases of recurring congestion (nonrecurring congestion will be examined below).

This shows up more clearly by examining the percentage of time saved or lost as the percentage of probes and the congestion level varies. For informed drivers and low congestion levels ( 0.5 or 0.67 volume to capacity ratios), probe information is simply too old to be of any value. Ignoring old probe reports will be better than listening to them. However, as traffic approaches and exceeds capacity, a critical mass of probes can be useful. As the share of probe vehicles on the network exceeds $30 \%$, the percentage of time saved for informed drivers becomes positive. Uninformed drivers also have benefits in congested conditions when probes comprise $30 \%$ or more of the fleet and even modest benefits for relatively uncongested conditions at a much higher share of probe vehicles ( $80 \%$ or more). Examining the standard deviation for informed and uninformed drivers, respectively, as the percentage probe and congestion level vary (at an average percentage informed of 50\%), the more probes on the network, the lower the standard deviation. The standard deviation does increase with congestion. Informed and uninformed 


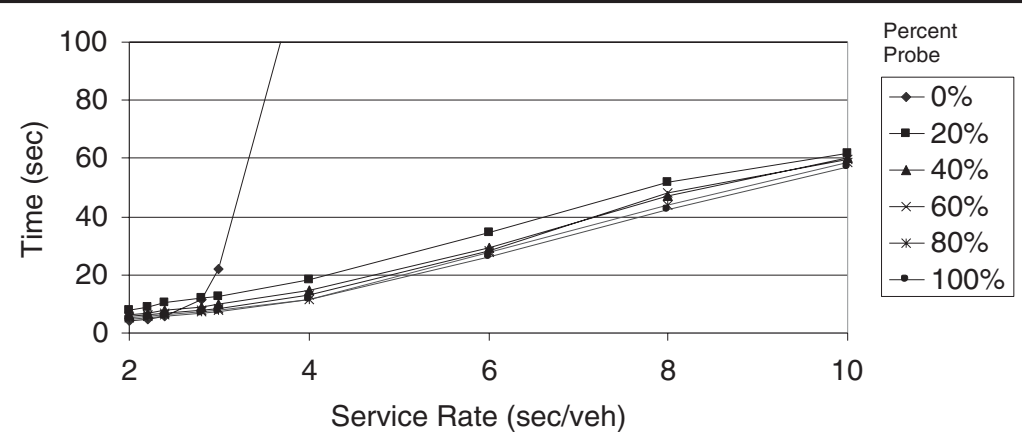

Figure 4: Time (Informed Travelers) by Service Rate and Percentage Probes

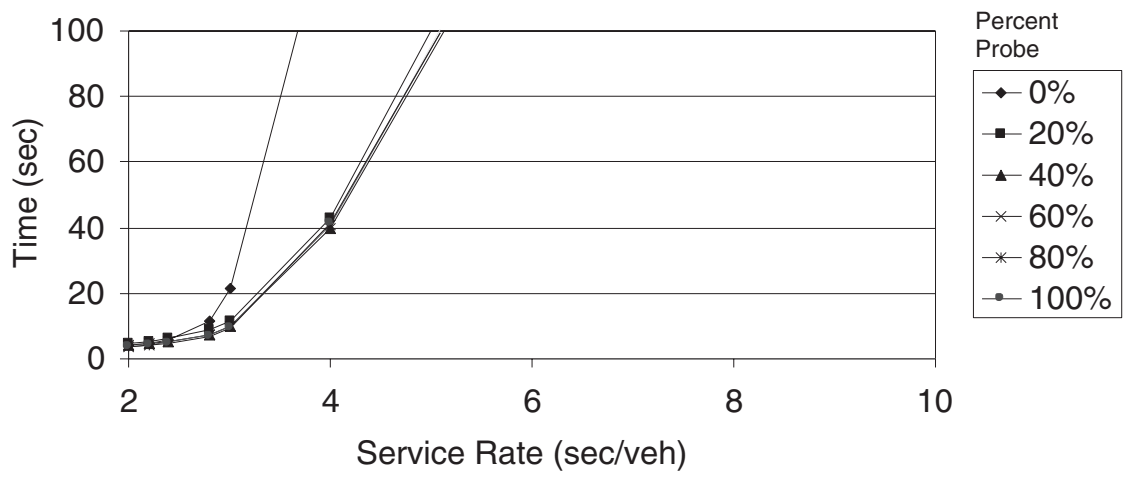

Figure 5: Time (Uninformed Travelers) by Service Rate and Percentage Probes

drivers have similar standard deviations, varying only at low percentage probes, where misinformation leads to informed drivers having a higher variance.

\section{NONRECURRING CONGESTION}

Although probes have some serious drawbacks in assisting travelers during recurring congestion, the same is not true of nonrecurring incidents. Here we consider the effects of information as the service rate increases on one link while a parallel link retains its capacity characteristics ( 2 seconds per vehicle). It is assumed that the capacity reduction is for the entire period (the time required to serve 1,000 vehicles arriving at 0.67 vehicles per second).

Examining the model when we unexpectedly vary service rates on one link (a surrogate for an incident), we see that as the time to serve each vehicle increases, the average time increases (see Figures 4 and 5). However, as the number of probes on the network increases, the average time tends to decrease. Even a few probes are sufficient to shift almost all informed travelers to the shortest route, although the more probes on the network, the faster an incident will be detected and the faster an overreaction can be arrested. Informed travelers can save a great deal of travel time because they completely avoid the incident (although the link without the incident may still have some congestion). Furthermore, uninformed drivers save time too, because the informed drivers do not exacerbate the queues on the affected link (the queue is half as long without the informed drivers) when they switch to the link without the incident. Before the incident, $50 \%$ of travelers used each link and 50\% were informed. After the incident, $75 \%$ will use the unaffected link (of whom $67 \%$ are informed) and $25 \%$ will use the affected link (of whom $0 \%$ are informed). Although $75 \%$ of total travel on one link will lead to delay, this is much less delay than associated with the affected link. In all cases, more probes are better for the reasons noted above, although most gains can be achieved with a relatively low probe percentage ( $20 \%$ 


\section{Still, only a relatively small probe percentage is necessary to achieve most of the gains in reliability associated with information for non-recurring congestion.}

or less may be sufficient). The standard deviation increases with the decrease in capacity but tends to decrease as the number of probes increases. Still, only a relatively small probe percentage is necessary to achieve most of the gains in reliability associated with information for nonrecurring congestion.

\section{Summary and Conclusions}

The private provision of traveler information, from data collection through distribution, is an idea worth pursuing. The local American Automobile Association (AAA), for instance, would seem an ideal organizational structure. We could imagine that service is provided as part of dues, where each user installs GPS and user-end communications, whereas the AAA (or a contractor) collects, warehouses, collates, and serves the information. This can skirt many of the institutional issues with government data collection that have been hampering real-time traffic information systems. For instance, AAA already provides supplementary information used in traveler information systems in Pennsylvania (SmartRoute 1997) and has participated in the Orlando TravTek and Chicago ADVANCE studies.

Information provision is probably best for what an individual will not know from routine behavior - random incidents and unfamiliar territory. The qualitative conclusion that incidents and the unexpected are where the greatest gains from traveler information are to be found reinforces the results from our simulations. Those models show that a low level of probes can provide useful information by rapidly detecting incidents, whereas a much greater number is needed to provide any gains from recurring congestion.

As the costs of electronics and communications decline while congestion fails to abate, the market for in-vehicle navigation and roadside communication devices will grow. However, the usefulness of those devices depends very much on the quality of data they employ. Today's public sector data collection efforts in most metropolitan areas are insufficient to fully realize the potential of Advanced Traveler Information Systems. Alternatives such as private or quasi-public information clubs may provide an alternative that will exploit Advanced Traveler Information Systems to their fullest.

\section{Notes}

1. Different providers of traveler information (public or private) may have different objectives and thus provide different information. Government information providers may choose to supply either a user optimal (UO) or a system optimal (SO) route to travelers. On first blush, it may seem that a welfare-maximizing jurisdiction should route people for the benefit of all, convincing people to engage in a socially optimal route. However, if that socially optimal route is noticeably inefficient for individual users, it likely will be ignored. Still, with the proper incentives to overcome the extra user time involved in engaging in a nonuser optimal solution, travelers may be willing to choose that route. These incentives may be providing the information for free or at a discount compared to a private sector supplier or even providing a cash rebate. However, if only a fraction of users switch from UO to SO routes, others will change their UO routes, and overall, no gain takes place. In contrast, private providers will likely provide users with user optimal routing information, or they will soon go out of business. We may see multiple information providers or the government providing free SO information while private firms charge for UO information.

2. Although the simulation assumes identical capacities in the absence of incidents, an incident only affects one of the parallel routes. Assumption of asymmetrical links will not alter the general conclusions but simply the point at which switching routes becomes worthwhile.

\section{References}

Adler, J. L., Blue, V. J., \& Wu, T. L. (1999). Assessing network and driver benefits from bi-objective in-vehicle route guidance. Paper presented in the 78th Transportation Research Board Annual Meeting.

Al-Deek, H. M., \& Kanafani, A. (1989). Some theoretical aspects of the benefits of en-route vehicle guidance (ERVG) (UCB-ITS-PRR-89-2). Richmond, CA: University of California at Berkeley PATH Program. 
Al-Deek, H. M., \& Kanafani, A. (1993). Modeling the benefits of advanced traveler information systems in corridors with incidents. Transportation Research, 101(4), 303-324.

Boyce, E. D., Hicks, J., \& Sen, A. (1991). In-vehicle navigation requirements for monitoring link travel times in a dynamic route guidance system. Operations Review, 8(1), 17-24.

Dallenback, S. W., \& Fariello, B. (2001). TransGuide(r) Model Deployment Initiative. Available: http://www. transguide.dot.state.tx.us/docs/transtec.html

Emmerink, R.H.M., Axhausen, K. W., \& Rietveld, P. (1995). Effects of information in road transport networks with recurrent congestion. Transportation, 22, 21-53.

Levinson, D., Gillen, D., \& Chang, E. (1999). Assessing the benefits and costs of intelligent transportation systems: The value of advanced traveler information systems. California PATH Research Report for California Department of Transportation UCB-ITS-PRR-99-20

Peckmann, M. (1996, October 14-18). STORM stuttgart transport operation by regional management—Results on the field-Trials and assessment. In the 3rd Annual World Congress on Intelligent Transport Systems: "Intelligent Transportation: Realizing the Future," Orange County Convention Center, Orlando, Florida, ITS America.

Rakha, H., \& Van Aerde, M. (1995, March 15-17). Accuracy of vehicle-probe estimates of link-travel time and instantaneous speed. Intelligent transportation: Serving the user through development: Proceedings of the 1995 Annual Meeting of ITS America, pp. 385-393.

Saricks, C. L., Shofer, J. L., Sööt, S., \& Belella, P. A. (1997). Evaluating effectiveness of real-time advanced traveler information system using a small test vehicle fleet. In Transportation Research Record 1588 (pp. 41-48). Washington, DC: TRB, National Research Council.

Sen, A., Thakuriah, P., Zhu, X. Q., \& Karr, A. (1997, July/August). Frequency of probe reports and variance of travel time estimates. Journal of Transportation Engineering, 123(4), 291-297.

SmartRoute 1997. Transportation Secretary Mallory and SmartRoute Systems, Inc. Launch New Traveler Information Service in Philadelphia Region. http://www.smartroute.com/press/pr-5-22-97.htm

U.S. Department of Transportation. (1995). TravTek evaluation Orlando test network study (Publication No. FHWARD-95-162). Washington, DC: Author.

Wunderlich, K. E. (1995, October). Congestion and route guidance benefits assessment. (Letter ITS-L-131). McLean, VA: The MITRE Corporation.

Wunderlich, K. E. (1996). An assessment of pre-trip and en route ATIS benefits in a simulated regional urban network. Presented at the 3rd World Congress on Intelligent Transport Systems, "Intelligent Transportation: Realizing the Future," Orlando, Florida. 